\title{
OPTICAL CHARACTER RECOGNITION: AN ENCOMPASSING
}

\section{REVIEW}

\author{
Nikhil Pai ${ }^{1}$, Vijaykumar S. Kolkure ${ }^{2}$ \\ ${ }^{I}$ M.E. (Electronics, Appeared), Department Of Electronics Engineering, Bharatratna Indira Gandhi College of \\ Engineering, Affiliated to Solapur University, Solapur, Maharashtra, India. \\ ${ }^{2}$ Assistant Professor, Department Of Electronics Engineering, Bharatratna Indira Gandhi College of Engineering, \\ Affiliated to Solapur University, Solapur, Maharashtra, India.
}

\begin{abstract}
Optical character recognition (OCR) is becoming a powerful tool in the field of Character Recognition, now a days. In the existing globalized environment, OCR can play a vital role in different application fields. Basically, OCR technique converts images into editable format. This technique converts images in the form of documents such as we can edit, modify and store data more safely for longtime. This paper presents basic of OCR technique with its components such as pre-processing, Feature Extraction, Classification, post-processing etc. There are various techniques have been implemented for the recognition of character. This Review also discusses different ideas implemented earlier for recognition of a character. This paper may act as a supportive material for those who wish to know about OCR.
\end{abstract}

Keywords- OCR, Feature Extraction

\section{INTRODUCTION}

Now a days, globalization is reaching to a great level. In this globalized environment, character recognition techniques also getting a valuable demand in number of application areas. OCR is an effective technique which converts image into suitable format such that data can be edit, modify and stored. This technique performs several operations such as, scans the input image, processes over the scanned image thereby image gets converted into portable formats .For instance, the hard copy of old historical books, novels, etc. .cannot be stored safely for a long time. Rather, its safety has limitations. If we apply OCR technique for such cases, the different historical documents can be stored, modified for a longtime. OCR also having variety of applications in almost all fields, including security. OCR implementation helps us to edit, store and process over the scanned data more effectively. User can handle the stored data whenever he wants with the internet support. So Optical character recognition is most successful application used in pattern recognition. A typical OCR system consists of the following basic components:

1. Input scanned Image

2. Pre-processing

3. Feature Extraction

4. Classification

5. Post- processing $* * *$

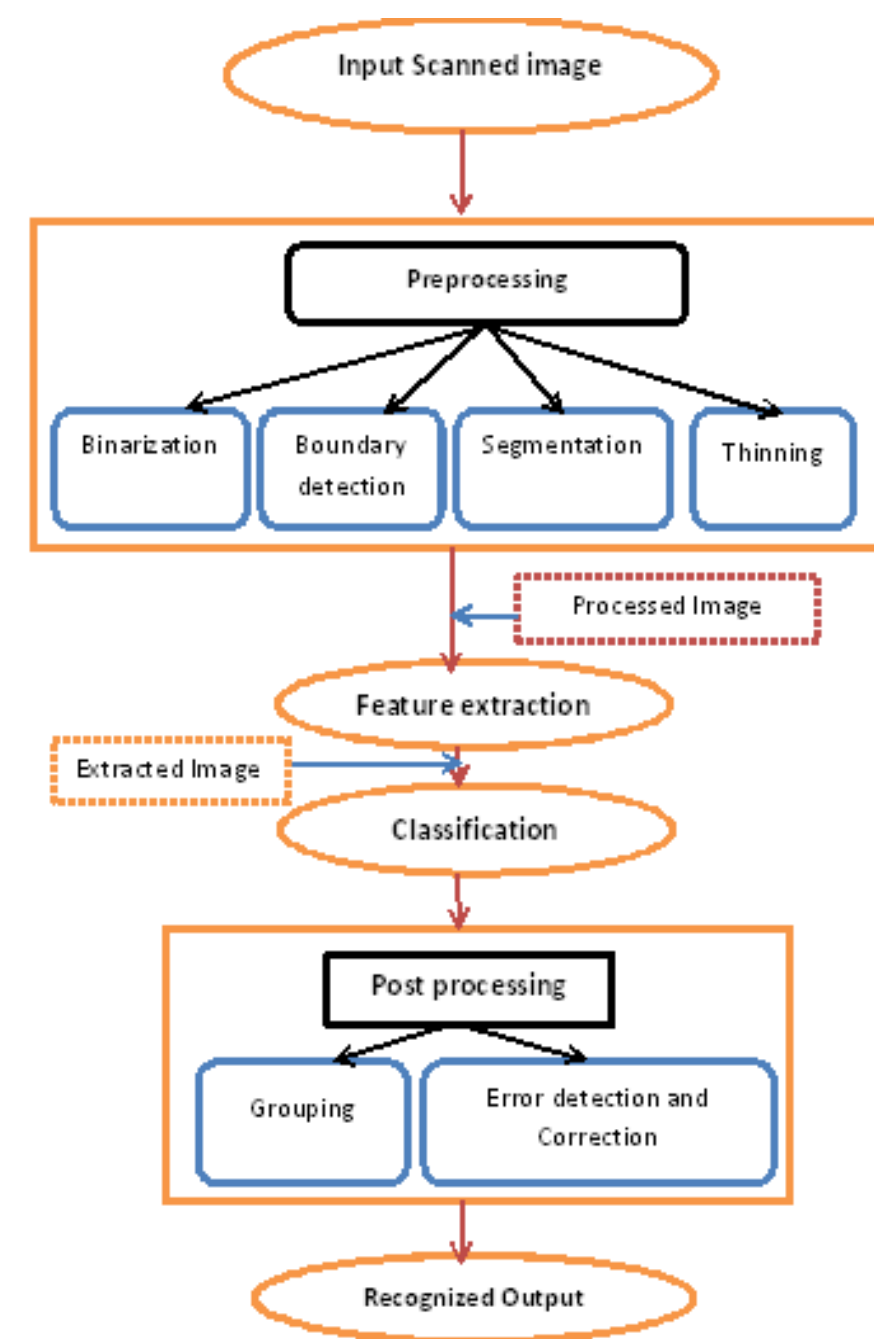

Fig- 1.1: Processing Stages of OCR Technique 


\section{Input Scanned Image}

Firstly, image of input data is optically scanned. The scanned image can be any document of different dimensions. This scanned input image is fed to pre-processing section so as to process over that scanned image.

\section{Pre-Processing}

Pre-processing includes several operations over the scanned image, so that input image becomes suitable and comfortable for applying to further sub sections. Basically the objective of pre-processing is to improve the quality of scanned input image. Noise removal, mathematical operations can also be processed in this Pre-processing section. It includes binarization, boundary detection, segmentation, thinning. It performs the several operations over the scanned input data.

\subsection{Binarization}

Binarization plays an important role in pre-processing. It is necessary to convert a color image into black and white format. So we can process over that black and white image. Basically separation of background and actual image area referred as foreground of a scanned image is called binarization.

\subsection{Boundary Detection}

The binarized image is now applicable for boundary detection. In this operation the boundaries of scanned image is detected. It detects all the boundaries of image. It is necessary to detect the boundaries so as to select an individual character.

\subsection{Segmentation}

This is important operation of OCR as rate of recognition is directly proportional to segmentation. In this process, every individual character is separated. This isolates the different sub-parts of an image. It is used to separate pixels of an image as per the contents in data like words, paragraph etc.

\subsection{Thinning}

Thinning is used to clean the scanned input image. This process deletes the dark points in the image.

\section{Feature Extraction}

For the accuracy of OCR system, the appropriate Feature Extraction method should be selected. While processing over the image some features should be separated. The typical features are Edges, Corners, Ridges, etc. This method of separation is called as Feature Extraction. The accuracy of an OCR technique depends on selection of proper feature extraction method.

\section{Classification}

The feature extracted data must have gone through the process of Classification. This process classifies the extracted individual character in proper way.

\section{Post-Processing}

This is the last and an important phase of OCR technique. It includes different operations like Grouping, Error detection and correction. Whatever the data being operated through different operations such as, binarization, segmentation, Feature extraction, Classification etc. is fed to postprocessing. That means different features of input scanned image are extracted. That feature extracted data is an individual character. It is unable to get detailed information from that individual character. So, it is necessary to collect individual character in appropriate and sequential manner. The process of collecting individual characters of the same contents to form a string is termed as Grouping. By using error detecting and correcting algorithms, errors can also be eliminated.

Finally, we get the recognized output character.

\section{LITERATURE REVIEW ON OPTICAL}

\section{CHARACTER RECOGNITION}

As per reference, IJETAE Volume 4, Issue 5, May 2014 ${ }^{[1]}$ this paper explains comparative analysis between Random Transform and Hough Transform, which are applied for error detection and correction. This paper explains implementation of OCR in Matlab, compared with current working method of OCR. This system achieved recognition rate near about $92 \%$.

As per reference, IJSR Publications, Volume 2, Issue 6, June $2012^{[2]}$ this paper discusses recognition of off-line English character. This explains a new model Hidden Markov Model (HMM) for character recognition. The Novel feature Extraction method is used for implementing HMM. By collecting 13000 samples from 100 writers they have tested performance of OCR technique and got accuracy of near about $94 \%$.

As per reference, IJARECE Volume 2, Issue 5, May $2013^{[3]}$ this paper implements the OCR technique in Matlab. This paper explains how matlab is more convenient and effective for OCR technique. The performance of OCR has been tested with samples in this approach.

As per reference, European Academic Research, Volume I, Issue 5/ August $2013^{[4]}$ this paper discusses the OCR technique with its components. This achieved a good recognition rate by implementing Particle Swarm Optimization Approach.

As per reference, International Journal of Research in Computer and Communication Technology, Volume 2, Issue 9, September $-2013^{[5]}$ This explains basic of OCR technique with its components including Pre-processing, Segmentation, Feature Extraction and Classification.

As per reference, Applied Computational Intelligence and Soft Computing Volume 2012, Article ID $897127^{\text {[6] This }}$ paper proposes comparative study of new ideas, Particle Swarm Optimization (PSO) and Bacterial Foraging 
Optimization (BFO). In this proposal PSO and BFO are used to achieve most advantageous harmonic compensation. This paper also discusses the efficiency of both approaches PSO and BFO by comparing them.

As per reference, IJERA Volume 1, Issue 4, pp. 1736-1739

[7] this paper presents an overview of the various O.C.R. systems for Gurumukhi which are developed for handwritten isolated Gurumukhi text. This paper discusses details of different feature extraction methods with its comparative analysis.

As per reference, International Journal of Advanced Research in Computer Science and Software Engineering Volume 4, Issue 1, January $2014^{[8]}$ this explains OCR technique for both handwritten and printed Guajarati script. For this implementation, linear recognition technique has been used. This paper explains how linear recognition technique is efficient in OCR for error detection and correction.

As per reference, International Journal of Computer Application, volume 23, no.1, pp. 21-24, 2011. ${ }^{[9]}$ This paper not only explain OCR for different font size and style, but also tests the performance of proposed OCR system with four groups of different font size and style. This proposed system achieved recognition rate near about $96 \%$.

\section{CONCLUSION}

This paper presents detailed description of OCR system. It includes discussion of various sub-parts of OCR technique such as, pre-processing, segmentation, Feature Extraction. The different papers having new algorithms and approaches so as to recognize a character accurately have been discussed in this review. Each technique has its own uniqueness and level of accuracy, but still some modifications have to be done for characters of different size and fonts.

\section{REFERENCES}

[1]. Jagruti Chandarana, Mayank Kapadia Optical Character Recognition International Journal of Emerging Technology and Advanced Engineering Volume 4, Issue 5, May 2014

[2]. Binod Kumar Prasad, GoutamSanyal A model Approach to Off-line English Character Recognition International Journal of Scientific and Research Publications, Volume 2, Issue 6, June 2012

[3]. Sandeep Tiwari, Shivangi Mishra, Priyank Bhatia, Praveen Km. Yadav[May 2013] Optical Character Recognition using MATLAB International Journal of Advanced Research in Electronics and Communication Engineering (IJARECE)Volume 2, Issue 5, May 2013

[4]. Majida Ali Abed Hamid Ali Abed Alasadi Simplifying Handwritten Characters Recognition Using a Particle Swarm Optimization Approach European Academic Research, Volume I, Issue 5/ August 2013

[5]. Mahesh Goyani, Harsh Dani, Chahna Dixit Handwritten Character Recognition - A Comprehensive Review International Journal of Research in Computer and
Communication Technology, Volume 2, Issue 9, September 2013

[6]. Sushree Sangita Patnaik and Anup Kumar Panda Particle Swarm Optimization and Bacterial Foraging Optimization Techniques for Optimal Current Harmonic Mitigation by Employing Active Power Filter Applied Computational Intelligence and Soft Computing Volume 2012, Article ID 897127.

[7]. Pritpal Singh, Sumit Budhiraja Feature Extraction and Classification Techniques in O.C.R. Systems for Handwritten Gurumukhi Script - A Survey International Journal of Engineering Research and Applications (IJERA) Volume 1, Issue 4, pp. 1736-1739.

[8]. Lipi Shah, Ripal Patel, Shreyal Patel, Jay Maniar Skew Detection and Correction for Gujarati Printed and Handwritten Character using Linear Regression International Journal of Advanced Research in Computer Science and Software Engineering Volume 4, Issue 1, January 2014

[9]. Ritesh Kapoor, Sonia Gupta, and C.M. Sharma, "Multifont/size character recognition and document scanning," Int. J. of Computer Application, volume 23, no.1, pp. 21-24, 2011.

\section{BIOGRAPHIES}

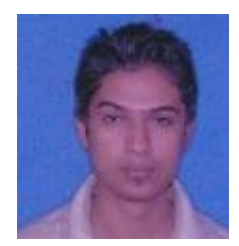

Nikhil Pai ${ }^{1}$ currently pursuing M.E. (Electronics) From Bharatratna Indira Gandhi College of Engineering, Solapur, Maharashtra, India. His area of interest is image processing, MATlab.

Vijaykumar S. Kolkure ${ }^{2}$ has completed M.E. (Electronics) from W.C. Sangli, Maharashtra, India. He has 10 years of teaching experience. Currently he is working as Assistant Professor at Bharatratna Indira Gandhi College of Engineering, Solapur, Maharashtra, India. His area of interest is Image Processing, Video Processing. 